\title{
Formación de usuarios de la información y procesos formativos: hacia una conceptuación*
}

\author{
Edilma Naranjo Vélez
}

Artículo recibido:

3 de mayo de 2004.

Artículo aceptado:

26 de octubre de 2004.

\footnotetext{
* Este trabajo forma parte de la investigación Evolución y tendencias de la formación de usuarios en un contexto latinoamericano, cofinanciada por el Centro de Investigaciones en Ciencia de la Información (CICINF) de la Escuela Interamericana de Bibliotecología de la Universidad de Antioquia y el Centro Universitario de Investigaciones Bibliotecológicas (CUIB) de la Universidad Nacional Autónoma de México, 2003.
}

\section{RESUMEN}

Alrededor de los usuarios de la información se tejen varias ideas y propuestas convertidas en teorías, definiciones, términos y conceptos que engrandecen la disciplina bibliotecológica. Se habla entonces de necesidades de información de los usuarios, características de los usuarios, perfil de usuarios, categorías y tipologías, estudios de usuarios, formación de usuarios. En esta ocasión el tema a tratar es la formación de usuarios, observando el concepto formación desde diferentes disciplinas y la relación que tiene con el concepto de usuario. También se presenta la evolución del término formación de usuarios en procura de un acercamiento a una conceptuación, pues leyendo la bibliografía que hay en el medio se advierte que alrededor del tema se ha escrito bastante, especialmente desde el campo metodológico. Es decir, se escriben propuestas de la forma cómo deben planearse, desarrollarse y, un poco menos, evaluarse los programas para la formación de 
usuarios, pero se observa muy poco el aspecto conceptual en que se apoyan al momento de elaborar las propuestas mencionadas. No es una labor terminada, es un inicio que se espera continuar trabajando para aportarle a la disciplina bibliotecológica.

Palabras clave: Formación de usuarios; Usuarios de la información.

\section{ABSTRACT}

Formation of information users and formative processes: towards a conceptualization

Edilma Naranjo-Vélez

Many ideas and proposals about users of information have been converted into theories, definitions, concepts, and terms, all of which enhance the library science discipline. User information needs, user characteristics, user profiles, categories and typologies, user studies, user formation, all come into consideration.

We take up user formation evolution and observe the term formation and its relationship with the concept of user from several disciplines, based on an exhaustive bibliography. We find that methodologically-speaking many approaches exist on how to plan and develop such proposals, but little support is available on the conceptual aspects. Obviously, this is not a finished task but only a beginning which hopefully will be carried on further.

Keywords: User formation; Information users.

\section{INTRODUCCIÓN}

in duda en esta época en la que se habla de la sociedad de la información, Olos miembros de la sociedad están buscando y produciendo más información en todos los medios, formas y soportes que les sea posible con el fin de lograr una comunicación que facilite la adquisición del conocimiento y el desempeño de la vida académica, laboral, profesional y cotidiana. Situación que implica que estas personas deben conocer y saber cómo encontrar la información y utilizarla de acuerdo con sus intereses y necesidades. 
Las unidades de información* procuran aumentar su validez cada vez más como las principales y mejores fuentes de información para los usuarios, y responder así a la misión y visión propuestas como sistemas al servicio de éstos.

Entre tanto las personas en búsqueda de información encuentran dificultades para acceder en forma efectiva y eficaz a ella, debido al todavía incipiente desarrollo de las habilidades y competencias que poseen y que se requieren para la búsqueda, localización, selección y evaluación de la información pertinente para resolver su necesidad en forma manual o con el uso de las tecnologías de la información y la comunicación (TIC) y así realizar las actividades anteriores, especialmente en momentos en los cuales se presenta un aumento permanente en la producción de nueva información.

Se necesita entonces que todo sujeto social adquiera y desarrolle habilidades, competencias y conocimientos que le permitan solucionar problemas y desempeñarse en su trasegar diario. Es de esta forma como la formación -concebida como un proceso que requiere un compromiso de todos los miembros de la sociedad- debe brindarse para que las personas, a partir de sus intereses y necesidades, aprendan, "para toda la vida", a detectar sus necesidades de información y a utilizar las TIC, lo cual les exige aprender a aprender y a aprehender esas habilidades, competencias y conocimientos para tener un acceso rápido y efectivo a la información pertinente, y por ello los bibliotecólogos están en la obligación de formar a los usuarios en el uso de las herramientas y en el desarrollo de habilidades de información que les permitan ser personas aptas en el acceso y el uso de la información.

Tratando de cumplir cabalmente con este papel los bibliotecarios han trabajado desde el siglo XIX expresando propuestas y reflexionado sobre su papel como formadores; sin embargo muy pocos han formulado un concepto que represente a este término que hace parte de la bibliotecología y la ciencia de la información. En este artículo se presenta el concepto usuario tratado desde la bibliotecología, al igual que las ideas formuladas por algunos teóricos sobre el concepto formación desde la filosofía y la pedagogía, y la evolución histórica de esta función, propia de las unidades de información. Elementos todos ellos que, unidos con los puntos de vista de expertos de la bibliotecología, permiten formular un concepto para la formación de usuarios que, a pesar de no considerarse concluido, se presta para comprender el sentido de éste y su uso dentro de la investigación que está desarrollando la autora del artículo; al final se presentan varias conclusiones como consideraciones finales de la reflexión.

* Para efectos de este trabajo, se utilizará el término unidades de información para referirse a todo tipo de bibliotecas (públicas, escolares, académicas y especializadas), centros de documentación, archivos y museos. 
El diccionario de la Real Academia Española, ${ }^{1}$ dice que usuario es quien usa ordinariamente una cosa. En el mundo de la información se encuentran personas tanto productoras como consumidoras de información, independientemente del lugar donde la encuentren y del soporte en que ésta esté presentada; a estas personas se les denomina usuarios de la información y aproximadamente desde la década de 1930 se les ha considerado como el centro y razón de ser de las unidades de información, a partir de la idea formulada por Louis Shores de que la "biblioteca debe estar centrada en el usuario". $^{2}$

Pensando en este sujeto, las unidades de información llevan a cabo procesos de organización de la información, desde la selección, adquisición y organización física de los recursos, hasta el diseño de programas de formación para que el usuario pueda hacer uso completo y adecuado de los servicios. Por eso se dice que él es el inicio y el final del ciclo de transferencia de la información. Además la infraestructura cognoscitiva que posee le permite ser un sujeto constructivo, creativo, sistemático y que generalmente busca orientación de tipo cualitativo ${ }^{3}$ en un sistema de información para interpretar y analizar la información, para entretenerse y para crearla o re-crearla.

Esta persona, que presenta un estado deficitario o anómalo del conocimiento, busca hacer uso de la información donde quiera que ésta se encuentre, no necesariamente en un sistema bibliotecario, puesto que su pretensión es modificar ese estado de conocimiento reflejado en una necesidad de información que procura satisfacer (en este sentido es fundamental aclarar que hay ocasiones en las que él no es consciente de esa necesidad de información o no sabe manifestarla); necesidad que puede estar relacionada con diversos problemas o intereses. La información que él consume, produce y brinda debe buscarla, solicitarla, analizarla, evaluarla y recrearla; el uso de la información le permite tomar decisiones relacionadas con su quehacer y estilo de vida; y, además, generar nuevas ideas, conocimientos y productos.

A pesar de que en épocas pretéritas fue un ser pasivo, en espera de que le dijeran cómo debía comportarse o qué debía hacer, hoy en día -en muchos lugares- participa activamente en el desarrollo de los sistemas de información y sugiere la creación o mejoramiento de los servicios de información, y

1 Real academia española. Diccionario de la lengua española. 2 ed. Madrid: Real Academia Española, 2001.

2 Richar Bopp y C. Smith. Introducción general al servicio de consulta : libro de texto para el estudiante de bibliotecología y manual para el bibliotecario de consulta / Trad. Carlos C. Compton García. México : UNAM, CUIB, 2000. cuándo y cómo aprender a orientarse. 
ayuda en la definición de políticas y productos de estos sistemas, puesto que como usuario acude regularmente a las unidades de información en búsqueda de los documentos que solucionen sus necesidades de información, por todo lo cual es un ser determinante para el quehacer del sistema de información y de la calidad de los servicios que se le ofrezcan. Esto es así porque el usuario usa, ofrece y genera información al interactuar con los componentes del sistema de información y con el ambiente en el cual funciona éste.

Finalmente es el usuario quien se enfrenta a una realidad social que vive día tras día y que le plantea problemas y retos de tipo cognoscitivo, social, político, económico, cultural, sociológico, psicológico y recreativo que generalmente está en condiciones de resolver, puesto que él busca solucionar sus necesidades de información de acuerdo con su mundo cotidiano de desempeño y de accionar; por eso y recordando a Michael Menou y Claire Guinchat, ${ }^{4}$ en las unidades de información se debe acudir frecuentemente a la pregunta “¿información para quién?" por encima de la interrogante “¿información para qué?”

Con esta concepción que se tiene del usuario de la información debe verse el concepto formación, para posteriormente unir ambos en uno solo y entrar en el término formación de usuarios.

\section{FORMACIÓN}

Formación (bildung) es una vieja palabra alemana [...] su aplicación, su contenido significativo y su peso como concepto central [...] desde hace largo tiempo se usaba casi exclusivamente por personas que se tenían como "cultas", es decir, por escritores y lectores. ${ }^{5}$

El término formación ha sido utilizado indistintamente para referirse a educación, instrucción u orientación; e inclusive en algunos casos ha sido relacionado directamente con la alfabetización. Sin embargo existen autores como Herder, Hegel, Honore, Rousseau y Gadamer, entre otros, que han tratado de darle explicación al término, y éste es tan abstracto que se usa en campos tan diversos como el de la estética (formación estética), la moral (formación moral), la literatura (formación literaria), la social (formación social), entre otras; de allí que se de la posibilidad de abstraerla al campo de la bibliotecología con los usuarios de la información.

4 Claire Guinchat y Michael Menou. Introducción general a las ciencias y técnicas de la información y documentación / Claire Guinchat y Michael Menou. 2. ed. cor. y aum. Por MarieFrance Blanquet. París : UNESCO, 1993.

5 Rudolf Vierhaus. "Formación = Bildung”/traducido por Juan Guillermo Gómez García, en Revista Educación y Pedagogía. Medellín. 2a época, Vol. 14 (May.-Ago., 2002); separata, p. 8. 
Juan Jacobo Rousseau ${ }^{6}$ dice que Formar es conocer la vida y el hombre, conocer la sociedad y los fenómenos culturales. Mientras que $\mathrm{Kant}^{7}$ dice que es el deber que cada uno tiene de darse forma; Humboldt ${ }^{8}$ lo amplía al decir que el proceso es continuo y nunca termina, pues se forma el lenguaje, la escritura, la moral y la ética. Para ello la casa, que implica protección, y la escuela, en tanto relacionada con la instrucción, cumplen un papel formativo, observado posteriormente en la educación y formación completa del sujeto, como parte del ciclo. Para Hegel ${ }^{9}$ es ascender a la generalidad, con una capacidad de abstracción en la que hay formación práctica y teórica y Herder ${ }^{10}$ dice que el concepto de formación se identifica con el concepto de cultura, que le da forma a las disposiciones y capacidades naturales del hombre.

Entendiendo la formación como una experiencia semejante entre hombres, cosas y sabiduría, Kant ${ }^{11}$ privilegia la experiencia de las cosas y de la naturaleza y se distancia de la experiencia de los hombres; con la explicación de Kant se ve cómo cada uno es responsable de sí mismo en su proceso formativo. Entre tanto, Gadamer ${ }^{12}$ dice que es apropiarse totalmente de lo que permite la formación a partir de la interacción, en la cual el sujeto como ser pensante es consciente de sus logros y apropiaciones, en un proceso constante de desarrollo y progreso, entonces la formación se asume como el conjunto de interacciones que vive el sujeto al entrar en contacto con el mundo.

Carlos Gallego y Nelly Villegas se refieren a "la aprehensión que el sujeto hace del mundo que lo rodea, propiciado por las relaciones que el mismo sujeto establece". ${ }^{13}$ Dentro de esa aprehensión participan la escuela, la religión, la política y el lenguaje como instituciones sociales que comparten la formación de los sujetos dentro del proceso de adquisición de cultura, como parte del patrimonio intangible del hombre. El lenguaje, considerado uno de los principales componentes en el proceso formativo que permite el diálogo, la comunicación e interacción con el otro, lo que facilita es la comprensión y apropiación de una cultura, un idioma y una idiosincrasia, aspectos esenciales del entorno que inciden en el proceso formativo integral del sujeto.

6 Humberto Quiceno Castrillón. "Rousseau y el concepto de Formación”, en Revista Educación y Pedagogía. Universidad de Antioquia. Medellín. No. 14-15 (1995); p. 87.

7 Immanuel Kant. Conflicto de las facultades. 1. ed. Buenos Aires : Losada, 1963.

8 Wilhelm von Humboldt. Sobre la diversidad de la estructura del lenguaje bumano y su influencia sobre el desarrollo espiritual de la bumanidad. Barcelona : Anthropos, 1990.

9 Guillermo Federico Hegel. Filosofía de la bistoria. Barcelona : Zeus, 1971.

10 Johann Gottfried von Herder. Ideas para una filosofía de la historia de la bumanidad. 1. ed. Buenos Aires : Losada, 1959.

11 Kant, Op. cit.

12 Hans-Georg Gadamer. Verdad y método. Salamanca : Ediciones Sígueme, 1991.

13 Carlos Gallego Escobar; Nelly Villegas Villegas y Diana Bernal Ocampo. Formación de lectores autónomos, un problema que trasciende a la escuela. Medellín : [S. n.], 1998; h 39. Tesis (Magister en Educación). Universidad de Antioquia, Facultad de Educación, 1998. 
Por lo tanto el hombre se apropia de los elementos del mundo, los reflexiona e introyecta, y establece sus propias relaciones al aprehender y acumular experiencias que le permiten ir adquiriendo la autonomía necesaria como sujeto social y pensante que toma decisiones avaladas por él mismo, al haber adquirido habilidades de pensamiento crítico y autónomo que le permiten estar en condiciones de abstraerse, producto de su cognición, tarea que requiere esfuerzo y sacrificio. De acuerdo con lo anterior y acudiendo a José León Crochik, puede decirse que:

La formación es la apropiación subjetiva de la cultura, y, por ello, implica la diferenciación, resultado de aquella apropiación. Es en este sentido como podemos entender que la constitución del individuo es mediatizada socialmente, y que cuanto más se aparta éste de la cultura en la búsqueda de sí mismo, menos se individualiza. La paradoja consiste en que cuanto más considera propias sus motivaciones, más está integrado a la sociedad que impone su autonomía.

\section{Luego,}

La formación es un proceso de desarrollo cuyo resultado se obtiene desde todas las influencias que con-forman al hombre, las cuales se encuentran revestidas, en algunas ocasiones, desde el proceso de enseñanza aprendizaje, en el que el hombre vive en una búsqueda continua de transformación, para lo cual es necesario que haga uso de la inteligencia de sí y del mundo; por eso se dice que es un ser inacabado, en continua trans-formación. ${ }^{14}$

Asimismo, José Alfredo Verdugo, ${ }^{15}$ desde la bibliotecología, explica que el concepto formación connota el proceso de desarrollo que permite manifestar al mundo exterior lo que es el mundo interior de cada sujeto, esto implica que en la formación se da un intercambio entre sujeto y contexto, contexto que le ha permitido vivir experiencias significativas y que le facilitan determinar lo que él quiere. En este proceso se da la oportunidad de intercambiar vivencias a partir de rupturas cognoscitivas. Por lo tanto, cuando la formación es planeada hace parte de un proyecto o programa en el que se da una instrucción sistemática, comúnmente llamada educación.

Con la formación se pretende orientar, instruir al sujeto en formación y, de ser posible, la transformación personal consciente del mismo, puesto que formación, cultura y educación modifican la vida social de los sujetos; esto

14 José León Crochik. La pseudoformación y la conciencia ilusionada, en Revista Educación y Pedagogía. Medellín. Vol. 12, no. 26-27 (Ene. - Ago., 2000); p. 159.

15 José Alfredo Verdugo Sánchez. « Hacia un concepto de formación de usuarios y propuesta de un programa”, en : Investigación Bibliotecológica : archivonomía, bibliotecología e información. México. Vol. 7, no. 15 (Jul. - Dic., 1993). 
es, el hombre debe prepararse para resolver problemas adquiriendo conocimientos, habilidades y destrezas, conforme a sus exigencias y características, lo que le permite producir el mayor rendimiento posible y origina la renovación o creación, puesto que él busca que sus saberes o conocimientos adquiridos se introyecten en su estructura cognoscitiva y por tanto también va en busca de la adquisición de formas o métodos de saber hacer.

\section{FORMACIÓN DE USUARIOS}

Examinados por separado los términos que conforman la expresión formación de usuarios, se procura entender ésta a la luz de la bibliotecología y la ciencia de la información las cuales se han apoyado en la psicología y la pedagogía para explicar cómo logra formarse al usuario de la información. Pero antes es necesario conocer las manifestaciones del mundo bibliotecario que han permitido llegar a formular la expresión formación de usuarios.

Historia del concepto ${ }^{16}$

El término formación de usuarios es uno de los utilizados para referirse al conjunto de actividades orientadas hacia el desarrollo de habilidades, competencias y conocimientos en los miembros de una sociedad para que logren hacer uso de la información en cualquier lugar donde ésta se encuentre y en cualquier formato y soporte. Es un término frecuentemente usado en América latina y Francia. Como dicen Miguel Ángel Arenas, Martha Elba Gutiérrez y Fausto Figueroa, ${ }^{17}$ con él el mundo bibliotecario se refiere "al conjunto de habilidades de aprendizaje permanente en relación con el conocimiento y uso óptimo de las fuentes de información o los recursos documentales para responder a necesidades específicas". Mientras que los anglosajones -de acuerdo con la traducción de los términos a la lengua española- hablan de "educación de usuarios" e "instrucción de usuarios" para denominar ese conjunto de habilidades de aprendizaje, que en otras ocasiones es denominado instrucción o educación en el uso de la biblioteca o se confunde con la instrucción bibliográfica.

Desde 1833 puede verse el interés de algunos bibliotecarios por resolver las dificultades existentes entre el usuario y la información; este interés se refleja

16 Parte de esta historia es adaptada de Richar Bopp y C. Smith. Introducción general al servicio de consulta : libro de texto para el estudiante de bibliotecología y manual para el bibliotecario de consulta / Trad. Carlos C. Compton García. México : UNAM, CUIB, 2000.

17 Miguel Ángel Arenas; Martha Elba Gutiérrez y Fausto Figueroa. "Uso de la información y su impacto educativo: la instrucción bibliográfica y su importancia”, en Reencuentro, núm. 21 (Mar. 1998), pp. 20-28. 
en el Informe Anual de la Universidad de Columbia, ${ }^{18}$ al sugerir en este documento el inicio de "una instrucción sistemática que inicie a los alumnos en los métodos correctos para que en el resto de sus vidas el trabajo en las bibliotecas pueda producirles el mayor rendimiento posible". 19 Ya en 1840 Emerson se pronuncia pidiendo que las universidades tengan un profesor de libros.

Aproximadamente en la década de 1850 las bibliotecas desarrollaron la formación de usuarios a la par con el servicio de consulta o referencia, debido a que los usuarios no se sentían con la capacidad de hacer uso de la biblioteca y sus servicios. La historia muestra que Samuel Green, en 1876, escribió un artículo que permitió comprender la importancia de los servicios de consulta para beneficiar a los usuarios, pero consideraba que ese beneficio sería real cuando se instruyera a los usuarios para buscar la información que necesitaban, especialmente en relación con el uso de las enciclopedias y los índices de los libros; en el mismo artículo también señalaba que las personas no sabían cómo utilizar con eficiencia una biblioteca. En ese año Dewey "afirmaba que había llegado el tiempo de que la biblioteca fuera una escuela, el bibliotecario un maestro y el visitante se convierta en un lector que utiliza los libros como los operarios sus herramientas". ${ }^{20}$

Teniendo en cuenta los datos anteriores que muestran el inicio de la formación de los usuarios, se establecen cuatro periodos para la historia de esta actividad, los cuales se encuentran organizados en los siguientes parrafos que destacan el papel de los tres Manifiestos de la UNESCO para la biblioteca pública y sus aspectos relacionados con la formación de los usuarios de la información.

Características desarrolladas de 1850 a 1920

- Los bibliotecarios manejaban conocimientos generales de alto rango, por eso desempeñaban el papel de docentes para atender a los usuarios; esto llevó a que en 1858 se les asignara el título de profesores de libros

- Por la profesionalización y especialización de los estudios académicos los bibliotecarios universitarios se dedicaron más a la selección del material bibliográfico y su respectivo análisis, y la formación se limitó a una charla sobre los libros y el uso de la biblioteca.

18 Richar Bopp y C. Smith. Introducción general al servicio de consulta : libro de texto para el estudiante de bibliotecología y manual para el bibliotecario de consulta / Trad. Carlos C. Compton García. México : UNAM, CUIB, 2000.

19 Rosario López de Prado. Información bibliográfica : [Documento en Internet] En : http://www.geocities.com/zaguan2000/414.html Última revisión: 24 de abril de 2000, [Consulta : 2003-11-13].

20 Ibidem. 
- El bibliotecario Azariah Root, quien trabajaba en el Oberlin College, desarrolló y dirigió un programa de instrucción de usuarios entre 1899 y 1927, en el cual les mostraba a los estudiantes los recursos de información, el sistema bibliotecario, y la historia de las obras impresas.

- Las bibliotecas públicas trataron de seguir la idea de Samuel Green y les dieron una atención personalizada a los usuarios para poder habilitarlos en el uso de la biblioteca y su información.

- En las primeras bibliotecas públicas de los Estados Unidos de Norteamérica programaban actividades para acercar a los usuarios a la lectura, y de esta manera les mostraban el valor y la importancia de la información para su desarrollo social, personal, cultural y económico.

- La mayoría de las bibliotecas públicas y universitarias limitaron la formación a una charla apoyada en un módulo de consulta o referencia para los diferentes grupos de usuarios, el cual hacía alusión a los recursos de información que la biblioteca poseía.

\section{Características desarrolladas de 1920 a 1979}

- Entre 1920 y 1930 los bibliotecarios se interesaron más por los usuarios miembros de una comunidad educativa y se dieron a la tarea de educarlos para que hicieran uso de la biblioteca, pues había empezado a interesar la expresión "la biblioteca centrada en el usuario", emitida por Louis Shores al crear el "Colegio de artes bibliotecarias".

- En 1940 Harvie Brans-Comb publicó la obra "Enseñando con libros", ésta sirvió para apoyar la integración de la biblioteca universitaria con la misión educativa de la universidad.

- La Royal Society of London Scientific en 1948 organizó la Conferencia sobre Información y en ésta se presentó la necesidad de diseñar cursos para el entrenamiento en el uso de la información para estudiantes universitarios. ${ }^{21}$

- Se publica el Primer Manifiesto UNESCO para la Biblioteca Pública en una época difícil para la humanidad, pues se estaba iniciando la postguerra y se veía a la educación como medio para sobrepasar los problemas de la sociedad; de allí que se pidiera a la biblioteca pública que completara la labor de la escuela para despertar el gusto por la lectura en niños y jóvenes y asegurara el aprecio y provecho de los libros en los adultos. Además se mencionaba la idea de darles consejos a los lectores sobre la manera de

21 Rocío Gómez Sustaíta. La enseñanza de las habilidades informativas. [Artículo en Internet] en http://www.uag.mx/Nexo/mar01/laense.htm, 2001. [Consulta: 2003-09-30] 
utilizar los libros y de ayudarles a decidir qué pensar, y para ello debían apoyarse en debates, conferencias, cursos o películas.

- En 1960 se le da a la biblioteca el carácter de centro de aprendizaje, concepto que tuvo bastante acogida y fue un factor de peso para que dentro de los currícula de la formación bibliotecaria se incluyera la formación de usuarios como materia clave, bajo los términos instrucción bibliotecaria, el lector y la biblioteca, entre otros. ${ }^{22}$

- Los movimientos sociales tuvieron gran influencia en el campo educativo, lo que también cobijó a las unidades de información. Los profesores no se limitaron al estudio de los libros sino que además utilizaron otras fuentes y soportes. También planearon la enseñanza de tal manera que el aprendizaje estuviera más centrado en la comprensión.

- Se da un gran apoyo a la investigación, lo cual exigió mejor preparación en el uso de las fuentes y la información.

- En América Latina la educación superior impulsó la formación de usuarios, como ocurrió con la Escuela Universitaria de Bibliotecología de la Universidad de la República en Uruguay, que desde 1945 realiza actividades de adiestramiento y formación de usuarios. En México también se dio un gran impulso a la formación de usuarios con la Facultad de Química Farmacéutica de la Universidad Nacional Autónoma de México; allí los bibliotecarios de la Dirección General de Bibliotecas y los profesores de la Facultad ofrecieron capacitación a los estudiantes en el uso del Chemical Abstracts, dada la complejidad del uso de este material bibliográfico. Con el tiempo y debido a su aceptación se convirtió en un curso obligatorio que entregaba nota aprobatoria y créditos dentro del plan de estudios. ${ }^{23}$ En 1967 la Universidad de las Américas ofrece el curso de introducción al uso de la biblioteca, curso con valor curricular y de carácter obligatorio. $^{24}$

- De igual manera, la Escuela de Medicina de la Universidad de Costa Rica empezó a ofrecer, en 1962, el curso de Bibliografía Médica (manejo de fuentes especializadas de información en medicina). ${ }^{25}$

- Se elabora y publica en 1972 el Segundo Manifiesto UNESCO para las Bibliotecas Públicas. Éste no hace una alusión tan directa al usuario y su formación; sin embargo, se rescata la propuesta en la que expresa que el fondo bibliográfico de la biblioteca al ser una prueba de la evolución del

22 Entrevista. Uriel Lozano Rivera, Luis Eduardo Villegas Puerta [Profesores Escuela Interamericana de Bibliotecología]. Entrevista elaborada por Yicel Giraldo G. y Edilma Naranjo V. Medellín, 26 de mayo de 2004. $120 \mathrm{~min}$.

23 Seminario Latinoamericano sobre formación de usuarios de la información y los estudios de usuarios / coord. Patricia Hernández Salazar. México : UNAM, Centro Universitario de Investigaciones Bibliotecológicas, 1997.

24 Ibid.

25 Ibid. 
saber y la cultura, ayudará a la gente a tener sus propias opiniones y desarrollar su capacidad de juicio, así como a ser críticos y creadores.

- En la década del 70 el trabajo de la "UNESCO a través del Programa UNISIST (United Nations Information System in Science and Technolo-gy) de Cooperación en el campo de la Información Científica y Tecnológica y del Programa General de Información (PGI) a usuarios de Ciencia y Tecnología; la Asociación Europea de Servicios de Información (EUSIDIC); la Federación Internacional de Documentación (FID) y la Organización de Cooperación y Desarrollo Económico (OCDE)", ${ }^{26}$ dejaron ver la importancia que se le estaba dando a la formación de los usuarios al programar seminarios y congresos bibliotecarios alrededor del tema.

- Una de las propuestas de UNISIST fue la creación de espacios y el intercambio de actividades para la discusión; así en 1976 se llevaron a cabo dos seminarios en Bangkok y en Roma.

- Se elaboraron instrumentos, directrices y publicaciones para aportar otros elementos en la formación de los usuarios; se recuerdan las Directrices para el planeamiento y la ejecución de un plan nacional de formación y enseñanza para el uso de la información.

- Se publicó en 1977 la guía para docentes del programa UNISIST, Educación y formación de usuarios de la información cientifica y tecnológica, preparada por Evans, Rhodes y Keenan.

- El apoyo que desde esas instituciones se dio a los países en desarrollo para capacitar a los bibliotecarios y que éstos a su vez capacitaron a los docentes, fue una muestra de la forma como se había metido en el medio el concepto de "la biblioteca centrada en el usuario".

- Autores como Wilson y Evans reflexionaron sobre el tema y dieron luces para elaborar las propuestas metodológicas de los cursos de formación de usuarios.

- Durante la década de los años 70, países como Argentina, Brasil, Cuba y Colombia incursionaron en la formación de usuarios de la información, como una medida que les permitiría a los estudiantes estar en condiciones de buscar y usar la información que requerían como futuros investigadores.

Características desarrolladas durante la década de 1980

- Se habla de instrucción bibliográfica y casi todas las bibliotecas apoyan este tipo de instrucción como un servicio de la biblioteca.

26 Stella Maris Fernández y Josefa Sabor. Formación de los usuarios de la formación educativa, 1987. p. 4. Citado por: Hernández Salazar, Formación en el uso de información : la instrucción computarizada como alternativa. México : [s. n.], 1996. Tesis [Maestra en Bibliotecología] Universidad Nacional Autónoma de México, Facultad de Filosofía y Letras. 
- El Consejo de la Asociación Bibliotecaria de Estados Unidos presentó una política en la que expresa que la instrucción bibliográfica debe iniciarse en los primeros años de la infancia y continuar en años avanzados para lograr así un usuario autónomo en la recuperación de la información, actividad esencial para su desempeño profesional. De igual manera, en 1980, el consejo afirma que 'es esencial que las bibliotecas de todos los tipos acepten la responsabilidad de proporcionar a la gente las oportunidades para entender y comprender la organización de la información. La declaración continúa y apremia a todas las bibliotecas 'para incluir la instrucción en el uso de las bibliotecas como una de las metas prioritarias del servicio'. ${ }^{27}$

- En 1982 se lleva a cabo la Reunión de Caracas y de allí surge la Declaración de Caracas, en la que se establecen los derroteros para crear y prestar servicios de información en América Latina; en esta Declaración se admite la importancia de formar un lector crítico, selectivo y creativo de la información, como manera de contar con un miembro activo de la sociedad. Además, considera de gran valía la creación variada de materiales de lectura que cubran los intereses de los usuarios de las bibliotecas.

- En Colombia se implementó la formación de usuarios en este periodo, con el desarrollo de un programa auto instructivo para usuarios de la información bajo el auspicio del ICFES (Instituto Colombiano para el Fomento de la Educación Superior). El programa fue ofrecido por bibliotecólogos, quienes se desplazaban hacia las diferentes ciudades del país y formaban a los docentes de las universidades, con el objetivo de que éstos replicaran en sus estudiantes lo aprendido.

- Durante ese periodo se inició intensamente el desarrollo y uso del término formación de usuarios en América latina.

- Se habla de la necesidad de evaluar los programas de formación de usuarios debido a los cambios ocurridos en la educación con los modelos pedagógicos de la década.

- Incursionan con gran aceptación las tecnologías de la información y logran que se incluya esta herramienta en la instrucción bibliográfica como recurso para recuperar información.

- En las Normas para Bibliotecas Universitarias promulgadas en 1986, la IFLA destaca el deber que tienen éstas de orientar sus servicios al usuario.

- La ALA (American Library Association) señala en 1989 que la biblioteca debe orientarse al futuro, asegurar la accesibilidad óptima de los servicios y realizar formación para el uso de recursos y servicios creados para los usuarios.

27 Carolyn A Kirkendall; Carla J. Stoffle. Instruction. En The service imperative for libraries : essays in honor of Margaret E. Monroe. Colorado : Libraries Unlimited, 1982; p. 42. Citado por: BOPP, Richar, SMITH, C. Introducción general al servicio de consulta : libro de texto para el estudiante de bibliotecología y manual para el bibliotecario de consulta. Trad. Carlos C. Compton García. México : UnAM, CUIB, 2000; p. 11. 
- La ALA presenta su definición sobre lo que se considera que es la alfabetización Informativa. ${ }^{28}$

Características desarrolladas hasta hoy

- Periodo caracterizado por la presencia de literatura profesional en el área y durante el cual se cubren aspectos teóricos, históricos y metodológicos de la formación de usuarios dados a conocer en eventos académicos como seminarios, congresos y reuniones, generalmente producto de las investigaciones desarrolladas por bibliotecólogos y cientistas de la información, como ocurre en América Latina con el Centro Universitario de Investigaciones Bibliotecológicas de la UNAM (CUIB), el Centro de Investigaciones en Ciencia de la Información (CICINF) de la Escuela Interamericana de Bibliotecología de la Universidad de Antioquia en Colombia, o con la Escuela de Bibliotecología y Ciencias de la Información en la Universidad de Costa Rica o el Centro de Investigaciones Bibliotecológicas de la Facultad de Filosofía y Letras de la Universidad de Buenos Aires (Argentina).

- Los bibliotecarios encargados de la formación de los usuarios han desarrollado nuevos conocimientos y habilidades para desempeñar su labor y respondido así a las exigencias de la sociedad, la economía y la política internacional.

- Las condiciones actuales de la sociedad rodeadas por las tecnologías de la información exigen que sus miembros desarrollen nuevas habilidades para acceder a la información y responder a las exigencias cambiantes del medio.

- Las unidades de información, en su mayoría automatizadas, deben formar a los usuarios en el uso de las herramientas y responder a sus inquietudes y necesidades tanto de información como de formación.

- Conceptos relacionados con la formación de usuarios, como desarrollo de habilidades informativas, alfabetización informacional o cultura informacional, empiezan a tomar fuerza en el ámbito bibliotecario y a enfatizar la presencia de las tecnologías de la información y la comunicación.

- En el Manifiesto de la UNESCO para Bibliotecas Públicas de 1994 se expone, en cuanto a la formación de usuarios apoyados con las tecnologías de la información y la comunicación en las bibliotecas, que: «habrán de establecerse programas de extensión y de formación de usuarios con objeto de ayudarles a sacar provecho de todos los recursos». ${ }^{29}$

28 American Library Association (ALA). Presidential Committee on Information Literacy. Final Report. (Chicago: American Library Association, 1989). [Documento en Internet] En : http://www.ala.org/acrl/nili/ilit1st.html [Consulta: 2004-08-30]

29 Edilma Naranjo Vélez y Nora Elena Rendón Giraldo. "Explorando el panorama de la formación de usuarios de la información”, en Revista Interamericana de Bibliotecología. Medellín. Vol. 26, no. 2 (Jul. - Dic., 2003); p. 20. 
- La Comisión Permanente de REBIUN, reunida en Girona (España) en 1997 formuló las Normas y Directrices para Bibliotecas Universitarias y Científicas, y estableció que la Biblioteca debe establecer sesiones de formación de usuarios en forma individual o colectiva, así como desarrollar programas para la formación continuada, pues se considera que el éxito de los programas de formación de los usuarios en las bibliotecas depende directamente de la integración de la biblioteca con los planes y programas de estudios de la universidad a partir de su función informativa y formativa.

- En la actualidad la biblioteca no sólo es un espacio físico, sino que se caracteriza por la prestación de servicios sin que sea necesaria la presencia física del usuario para que se beneficie de éstos.

- El PGI (Programa General de Información) de la UNESCO, adoptó en 1998 el Manifiesto de la Biblioteca Escolar UNESCO/IFLA; éste fue aprobado en la 30 Conferencia General IFLA en 1999 y en él se presenta una relación directa entre docentes y bibliotecarios para el mejoramiento de la lectura y la escritura, al igual que en el uso de las tecnologías de la información para lograr la alfabetización, el acceso a la información y, a largo plazo, el desarrollo económico, social y cultural de las naciones.

- En consonancia con el Manifiesto de la UNESCO para las Bibliotecas Públicas, se presenta en 1999 la Declaración de la IFLA sobre las bibliotecas y la libertad intelectual, en la que se muestra a estas unidades de información como los espacios que permiten el acceso al conocimiento, al pensamiento y a la cultura; además de ser un apoyo esencial para el aprendizaje a lo largo de toda la vida y permitir la toma de decisiones en forma autónoma, ${ }^{30}$ aspectos que hacen parte de lo que se denomina formación de usuarios.

- En el Tercer Encuentro sobre Desarrollo de Habilidades Informativas, realizado en Ciudad Juárez, México, en 2002, se presenta la Declaratoria de las Normas sobre Alfabetización Informativa en Educación Superior, con las cuales se busca facilitar el desarrollo de las habilidades informativas en los estudiantes universitarios y así fortalecer "su capacidad para acceder, evaluar e incorporar la información que requieren en sus procesos de construcción del conocimiento". 31

- Actualmente cada unidad de información de los diferentes países elabora sus programas de formación de usuarios haciendo uso o enseñando a utilizar

30 IFLA/FAIFE. Declaración de la IFLA sobre las bibliotecas y la libertad intelectual. La Haya: IFLA : FAIFE, 1999.

31 Normas sobre alfabetización informativa en educación superior : declaratoria. Jesús Cortés... [et al.]. En Tercer Encuentro sobre Desarrollo de Habilidades Informativas. Ciudad Juárez, 2002 (segundo borrador). Disponible en Internet en http://www.nclis.gov/libinter/infolitconf\&meet/JesusLauInfolitArticle-Spanish.pdf [Consulta : 2003-11-05] 
las tecnologías de la información y la comunicación; muchos de estos programas están publicados en la Internet.

Se ha presentado en forma muy sucinta una parte de la historia de la formación de usuarios; sin embargo, esto no es suficiente para llegar a entender las características que validan la existencia de este programa (llamado así por algunos), otros lo denominan función de las unidades de información; por ello y con el fin de dejar ideas más claras al respecto, se intentará definir y ahondar más en este concepto.

\section{Caracterizando la formación de usuarios}

Para centrarse en la conceptuación de la formación de usuarios de la información ya se vio, desde la pedagogía y la filosofía, el concepto de usuario de la información y el de formación; ahora se acude a la pedagogía y a la psicología educativa, especialmente a la primera, para caracterizar la formación de usuarios al observar al hombre como un ser en desarrollo y formación permanente tanto en el campo intelectual como en el espiritual y el afectivo; formación que se logra desde el interior mismo del sujeto, quien se muestra como un ser autónomo y libre que cultiva la razón y la sensibilidad, influenciadas por la cultura, la sociedad y el aprendizaje (aspectos propios de la psicología). De allí que haya una identificación con Rafael Flórez Ochoa cuando dice que:

Los conocimientos, aprendizajes y habilidades son apenas medios para formarse como ser espiritual. La formación es lo que queda, es el fin perdurable; [...] el hombre no es lo que debe ser como decía Hegel, y por eso la condición de la existencia humana temporal es formarse, integrarse, convertirse en un ser espiritual capaz de romper con lo inmediato y lo particular, y ascender a la universalidad a través del trabajo y de la reflexión filosófica, partiendo de las propias raíces. ${ }^{32}$

Continuando con Flórez Ochoa ${ }^{33}$ se puede afirmar que:

"Formar, pues, a un individuo en su estructura más general es facilitarle que asuma en su vida su propia dirección racional, reconociendo fraternalmente a sus semejantes el mismo derecho y la misma dignidad".

Por eso es una tarea bibliotecaria que debe verse como un proceso a largo plazo, y mucho mejor si se hace en forma ininterrumpida, para lo cual es

32 Rafael Flórez Ochoa. Hacia una pedagogía del conocimiento. Santafé de Bogotá : McGraw-

33 Ibid.; p, 111. 
fundamental la actitud asumida por el usuario; esta última característica hace que el proceso se considere optativo y de allí que para muchos no sea una condición necesaria.

Razón de más para que se diga que:

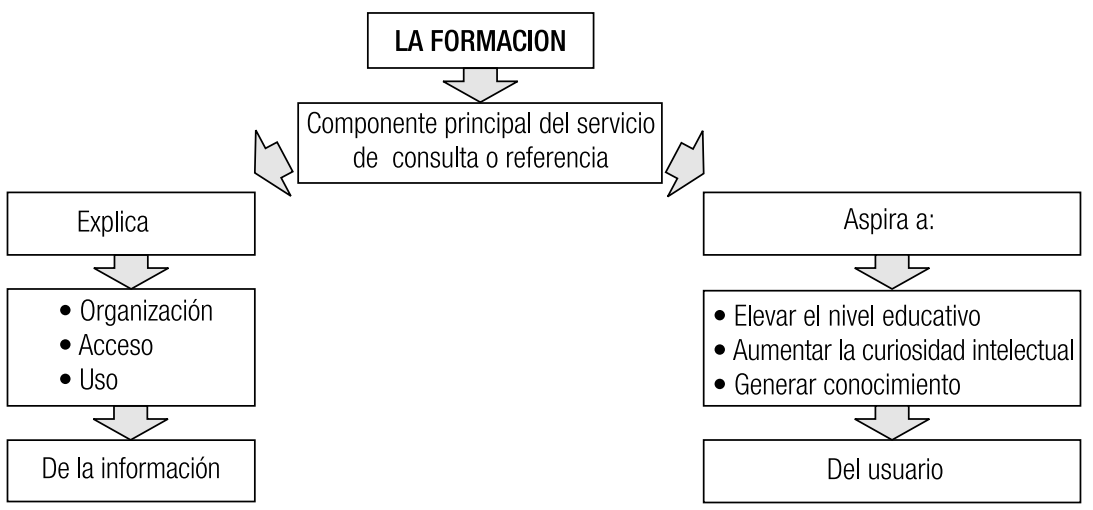

Asimismo se aspira a que las personas lleguen a actuar en forma autónoma y estratégica dentro de cualquier unidad y servicio de información, puesto que en el transcurso de su vida se enfrentarán a situaciones y contextos cambiantes.

Es recomendable que antes de abordar la planeación y el diseño de un programa de formación de usuarios se lleve a cabo un estudio de usuarios, en el que se analice el comportamiento de estas personas desde el momento en que hacen su ingreso a la unidad de información -si realizan una visita al espacio físico-, y se incluya además la necesidad de información, la manera cómo expresan su necesidad de información y cómo ejecutan el proceso de búsqueda de información, bien sea haciendo uso de los medios convencionales o los electrónicos; esto es, se identifican las características del individuo, relacionadas con el uso de información y su aprehensión del conocimiento (necesidades de información, estructura cognoscitiva, nivel y tiempo de experiencia en la disciplina de interés y acervo cognoscitivo).

Pues como dice Bernard Honore la formación es un proceso

“[...] por medio del cual se busca, en el otro, [profesor, instructor, coordinador del aprendizaje] las condiciones para que un saber recibido del exterior, luego interiorizado, pueda ser superado y exteriorizado de nuevo, bajo una nueva forma, enriquecido, con significado en una actividad". 34

34 Bernard Honore. Para una teoría de la formación: dinámica de la formatividad. Madrid: Narcea, 1980. 
Se pretende que los usuarios presenten una actitud positiva para aprovechar, tanto sus propios recursos mentales -que les permitan autorregular el aprendizaje-, como las herramientas tecnológicas. Por eso dentro del proceso de formación, el usuario debe ser preparado para mejorar las habilidades básicas y manejar las técnicas que le permitirán realizar un proceso de búsqueda completo y acorde con sus carencias cognoscitivas. También se busca desarrollar capacidades para que ellos reconozcan una necesidad de información, definan el tema y formulen correctamente la demanda informativa.

Cuando el usuario ha recibido una formación que ha tenido significado para él, es más factible obtener -en la unidad de información- un uso óptimo tanto de los servicios y recursos como de la información en general. De igual manera se pueden crear situaciones de comunicación con ellos, ya que pueden considerarse personas informadas y críticas en cuanto a las habilidades requeridas para desenvolverse en una unidad de información. De acuerdo con lo que dice Lhotellier la formación:

"es la capacidad de transformar en experiencia significativa los acontecimientos cotidianos generalmente sufridos, en el horizonte de un proyecto personal y colectivo [...] No es algo que se posee, sino una aptitud, o una función que se cultiva, y puede eventualmente desarrollarse". ${ }^{35}$

La formación de los usuarios facilitará en éstos la utilización eficaz de los servicios de información y la evaluación de estos servicios, y le permitirá localizar, organizar, utilizar, evaluar, interpretar y crear (comunicar) nueva información y, en lo posible, entretenerse con ella. ${ }^{36}$

Pero para lograr lo anterior es fundamental que el usuario adquiera conciencia sobre la importancia que tienen:

- La información para el desempeño de su vida en general.

- El reconocimiento de las necesidades de información.

- La expresión correcta de su demanda.

- Adquirir o desarrollar habilidades y destrezas dentro del proceso de búsqueda de información.

- Tener una actitud y una aptitud positiva.

35 Flórez Ochoa. Op. cit.

36 Esta apreciación se presenta al tener en consideración la idea que expone Posselt frente a la formación, éste dice que "La formación externa del hombre [...] sin una forma interior es simple brillo; la formación del gusto sin la formación del corazón es simple cultura; la formación del entendimiento sin la formación del corazón y del gusto es simple ilustración". Posselt, Franz. Apodemik, oder, die kunst zu reisen. Ein systematischer versuch zum Gebrauch junger reisenden aus den gebildeten ständen überhaupt und angehender gelerhten und künstler inbesondere. Leipzig : s. n., 1795. Tomo 1. Citado por: Rudolf Vierhaus. Op. cit.; p. 22. 
Esto hace alusión a una formación práctica, pues el hombre busca una satisfacción para sus necesidades e intereses de información y formación, para lo cual es fundamental que él sepa reconocerlos y expresarlos, acto que se ejecuta con los aprendizajes obtenidos durante la formación como usuario libre y autónomo, y tras el cual logra, idealmente, formular una necesidad de información.

Es importante ver cómo la UNESCO, por medio de Tocatlian -en la década de 1970 - presenta su propia definición del concepto; sin embargo, la definición involucra tanto la educación como la formación del usuario de una misma manera (es genérico): “[...] se entiende por 'educación' la actividad destinada a inculcar valores, actitudes y motivación, en tanto que la 'formación' consiste en la capacitación práctica”, ${ }^{37}$ y concreta expresando lo siguiente:

La educación y formación de usuarios incluye todo proyecto o programa destinado a orientar e instruir a los usuarios actuales y potenciales, individual o colectivamente, con el objetivo de facilitar: a) el reconocimiento de sus propias necesidades de información; b) la formulación de estas necesidades; c) la utilización efectiva y eficaz de los servicios de información, así como d) la evaluación de estos servicios. $^{38}$

Ante esta apreciación José Alfredo Verdugo Sánchez" aclara: "la educación se refiere al carácter y a la acción. La formación abarca la inteligencia de sí y del mundo que tiene el hombre".

Sin embargo, ahondando y ampliando más el término, es importante ver que la función de la formación de usuarios no debe limitarse a una planeación desde el punto de vista de la unidad de información, sino que se vuelve fundamental ver qué tan importante es el programa para el usuario, pues es en él en quien se está pensando, y es a partir de él que debe formularse la formación. Si se tienen en cuenta estos aspectos es posible lograr que el usuario alcance un aprendizaje significativo, puesto que un programa de formación de usuarios debe tener la intención de desarrollar en el usuario la capacidad para resolver problemas de tipo informativo con una actitud crítica, analítica y reflexiva para el manejo integral de la información, lo cual exige que él esté en condiciones de determinar qué información es relevante y pertinente y de incorporar la información nueva en su estructura cognoscitiva de un modo significativo. A este respecto nos dice Patricia Hernández que la formación de usuarios es un:

37 Jacques Tocatlian. "Formación de usuarios de la información: programas, problemas y perspectivas", en Boletín de la Unesco para las Bibliotecas. París. Vol. 32, no. 6 (Nov.-Dic., 1978). NOTA: El subrayado es nuestro.

38 Ibid.

39 Verdugo Sánchez. Op. cit. 
"proceso de intercambio de experiencias o saberes significativos sobre el uso de la información, con el fin de que la persona que la usa, de acuerdo con su proceso cognoscitivo pueda cambiar su actitud y aptitud en forma positiva, y optimizar dicha utilización al máximo". ${ }^{40}$

Además de lo anterior, para acometer la formación de usuarios es indispensable contar con un profesional de la información que muestre una actitud positiva hacia el usuario y sepa qué va a enseñar, a quién va a formar, y para qué y por qué va a formar, al usuario. Por lo tanto se necesita que los contenidos y exigencias sean acordes con las necesidades, intereses y motivaciones relacionadas con la información y la formación de los usuarios, pues lo que se busca es un intercambio real de experiencias significativas sobre el uso de la información, lo cual hará que el programa sea horizontal y flexible.

La formación de los usuarios es un programa que realza más el proceso que el resultado y que se ofrece por niveles, ${ }^{41}$ entre los cuales la orientación ${ }^{42}$ y la instrucción ${ }^{43}$ son los más reconocidos; y éstos se establecen de acuerdo con el tipo de usuario beneficiario ${ }^{44}$ y el tipo de unidad de información; asimismo se consideran los métodos, las técnicas, las estrategias de aprendizaje, la evaluación y los medios manuales y tecnológicos (herramientas).

El usuario se forma teniendo en cuenta ciertos objetivos de aprendizaje que buscan que éste desarrolle habilidades, competencias, conocimientos y actitudes ante el uso de fuentes impresas y electrónicas como recurso estratégico para el autoaprendizaje, y pueda utilizar las tecnologías que dan acceso a la información. Dadas las condiciones actuales relacionadas con la presencia de estas tecnologías, es necesario crear conciencia en los usuarios sobre el valor social de la información y las condiciones modernas de ésta; por lo tanto, deben proporcionársele al usuario los conocimientos necesarios sobre cómo

40 Hernández Salazar. La formación de usuarios de la información en instituciones de educación superior. México : UNAM, Centro Universitario de Investigaciones Bibliotecológicas, 1998.

41 Para entender esta expresión, de acuerdo con el Diccionario de las ciencias de la educación (Madrid : Santillana, 1983) nivel se refiere a la "determinación de exigencia en un determinado campo, tarea o facultad, que se precisa y detalla para poder cuantificar y comparar los resultados". Por esa misma línea, el diccionario expresa en relación a nivel educativo [formativo]: "Toda idea de nivel lleva a separación de categorías jerarquizadas, de acuerdo con determinados criterios".

42 Entiéndase por orientación el proceso de dirigir y guiar a las personas en el camino que les conviene seguir para obtener una información específica.

43 Proceso por el cual se desarrollan habilidades y competencias en los usuarios de la información para que busquen las fuentes de información pertinentes a su necesidad de información, y que las organicen de tal manera que les permita utilizar la información. Igualmente, se dice que se trata de una habilitación básica en el uso de una herramienta específica para que el usuario inicie y avance en la búsqueda, y de ese modo pueda interactuar con cualquier sistema y en cualquier unidad de información, independientemente de la profesión o labor que desempeñe o el nivel escolar en que se encuentre.

44 Dato que se obtiene, regularmente de los perfiles de usuarios que poseen las unidades de información. 
aprovechar los recursos de información y cómo funcionan los modernos medios de información documental.

Se da la formación para que el usuario comprenda el papel que tienen los servicios de información y referencia y el que cumplen otras instituciones del ámbito local, nacional e internacional para darle solución a las necesidades de información; por eso se recomienda que la formación se adecue a situaciones concretas que coincidan con los intereses del usuario. La formación de usuarios, además de ameritar la reflexión teórica y conceptual, requiere que los programas que se ofrecen estén sistematizados y se tenga una comprensión cabal del proceso que pretende llevarse a cabo y poder evaluar su efectividad.

En definitiva, frente a la formación del usuario puede decirse, avalando lo expuesto por María de Fátima Simôes, ${ }^{45}$ que:

[...] Para enseñarle algo será necesario hacerlo actor protagonista de una situación práctica, en la que pueda aprender por la propia experiencia. Otro principio importante se refiere a la idea de que para haber verdadera adquisición de conocimiento será necesario involucrar y llamar no sólo a la parte intelectual del aprendiz, sino a su totalidad psíquica. Una efectiva asimilación de conocimiento implica siempre una aplicación total, no sólo de la razón sino también de los afectos. Si el aprendiz no es absorbido por entero, el conocimiento permanecerá, por así decir, siempre exterior a él.

Puede verse entonces que la formación de usuarios se ofrece bajo varias modalidades y responden a la diversidad de unidades de información existentes, así:

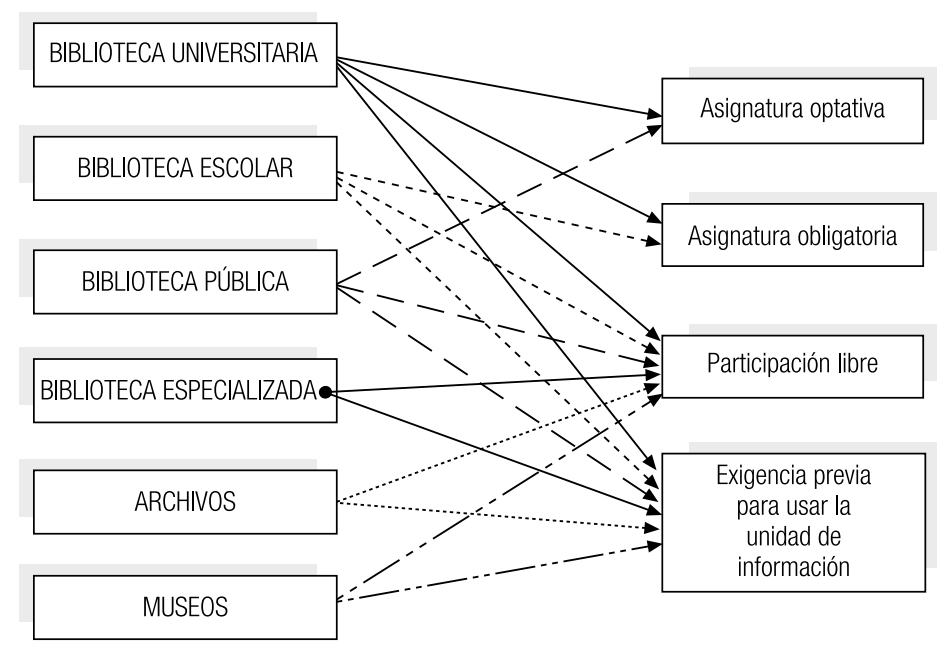

45 Francisco Simôes y María de Fátima. "La filosofía de la educación de Rousseau. Una propuesta de relectura del Emilio", en Revista Educación y Pedagogía. Medellín. Vol. 12, no. 26-27 (Ene. - Ago., 2000); p. 72. 
Independientemente de la modalidad en que se ofrezca la formación de usuarios, con ésta se busca:

- Aumentar la conciencia sobre el valor que tienen los recursos informativos.

- Apoyar todo el sistema de educación en los diferentes niveles educativos, para lo cual se considera a la formación de usuarios como el punto clave, el cual se inicia en la educación primaria y secundaria, bases de la educación superior.

- Acompañar la investigación científica y tecnológica, puesto que su desarrollo reposa en gran medida en el acceso a la información. Lo cual requiere del apoyo de los responsables de la investigación en el ámbito institucional y gubernamental.

- Facilitar la práctica de los usuarios en cuanto al acceso, localización, evaluación, selección, uso y producción de la información, desde los campos de desempeño de éstos, para que el aprendizaje sea significativo.

- Formar al ciudadano en general en el acceso a la información para y desde la vida cotidiana.

Lo anterior responde a una serie de preguntas que deben ser el insumo del programa de formación de usuarios que se pretende ofrecer. Algunas de estas preguntas son: ${ }^{46}$

1. ¿A quién va dirigida la formación? Respuesta que se logra con un estudio de necesidades de los usuarios.

2. ¿Por qué es necesaria la formación? El estudio mostrará la dimensión de las necesidades de los usuarios.

3. ¿Qué intenta lograr el programa de formación de usuarios? Con el estudio se establecen objetivos realistas para los programas.

4. ¿Dónde debe impartirse la formación? Todo depende de las características y categorías de los usuarios.

5. ¿Qué métodos de formación deben utilizarse? De acuerdo con las necesidades de los usuarios, se establecen los métodos.

6. ¿Cuándo debe brindarse la formación? Para su efectividad, se recomienda hacerlo en los horarios y ciclos de trabajo que hacen parte de la vida cotidiana de los usuarios.

Analizadas las características anteriores y las diversas definiciones presentadas por filósofos, pedagogos y bibliotecólogos, puede decirse que 
La formación de usuarios debe entenderse como un proceso ${ }^{47}$ que incluye un conjunto de acciones continuas, entre las que se encuentra el intercambio de experiencias y conocimientos, encaminados a la transformación permanente del usuario a partir de la comunicación de saberes sobre el uso de la información. Como proceso presenta una metodología propia y ofrece una serie de actividades de aprendizaje que requieren, por parte del formador, conocimiento sobre las actividades pedagógicas que le dan salida al proceso de enseñanza y aprendizaje, para conseguir así que el usuario domine las competencias y habilidades que le permitan formular su necesidad de información, así como buscar, localizar, seleccionar, analizar, evaluar y usar la información para su posterior transformación y comunicación. Asimismo, se busca que el usuario esté en condiciones de hacer uso de los servicios de una unidad de información y participar, de ser posible, en la evaluación y planeación de los mismos.

\section{Conclusiones}

La formación de usuarios es un proceso con el que se busca beneficiar al usuario para que esté en condiciones de solucionar sus necesidades de información. Además, sus diferentes actividades, programadas en forma paulatina y gradual, llevan al usuario por el mundo de la información; es decir, por la búsqueda, localización, recuperación, evaluación, uso y comunicación de la misma, como sujeto social que está en condiciones de ser autónomo en los aspectos que él se propone y que la sociedad le exige.

Las diferentes manifestaciones y declaraciones que ha formulado la comunidad bibliotecaria a lo largo del tiempo le han dado un lugar preponderante al usuario - con excepción del Manifiesto de la UNESCO para Bibliotecas Públicas de 1972. Sin embargo algunos de estos documentos lo ha denominado indistintamente lector, usuario y hasta cliente; términos que aparentemente son sinónimos pero que presentan delicadas diferencias que ameritan una investigación profunda para establecer una sustentada y valiosa discusión.

La expresión "la biblioteca centrada en el usuario", utilizada en la década de 1930, tiene hoy en día más peso, y en el caso de la formación de usuarios se observan propuestas formativas en las que se habla del aprendizaje significativo, producto de la validez que se le da a los conocimientos y experiencias previas que cada usuario posee al momento de iniciar su formación y durante el desarrollo de ésta. Idea que se refuerza con la pregunta formulada por Guinchat y Menou: “¿información para quién?”

47 Entendiendo por proceso: "una serie de actos u operaciones que conducen a un fin determinado." 
La formación de usuarios de la información debe planear la enseñanza que va a brindarle a los usuarios, y enfocarse a que éstos logren el aprendizaje trazado en los objetivos planteados. Por eso los conceptos, procedimientos y actitudes relacionados con el acceso y el uso de la información, deben articularse en torno a los conceptos de pensamiento, información y valores para obtener del usuario una actitud participativa y solidaria, en la que él se sienta involucrado y reconocido como sujeto social que tiene conocimientos previos que son de gran valía.

El proceso formativo pretende que el usuario sea autónomo, razón de más para ver la importancia que tiene este proceso al facilitar la localización, selección, procesamiento y comunicación de información, habilidades que exigen el desarrollo previo de otras, como son: observación, comparación, análisis de datos, comprensión, ordenación, clasificación y representación de fenómenos; es decir, habilidades de información unidas a las habilidades de búsqueda en la biblioteca.

La formación de usuarios ha sido una función de la biblioteca que ha tratado de ofrecerse desde tiempos lejanos y que aún sigue preocupando al mundo bibliotecario, sobre todo ahora que se incrementa la presencia de las tecnologías de información y comunicación, lo cual nos obliga a continuar profundizando en el tema de mejorar y facilitar la labor de los formadores de usuarios de acuerdo con los cambios que exige el mundo actual.

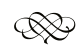

\section{Bibliografía}

American Library Association. Presidential Committee on Information Literacy. Final Report. (Chicago: American Library Association, 1989). [Documento en Internet] en:

http://www.ala.org/acrl/nili/ilit1st.html [Consulta: 2004-08-30]

Arenas, Miguel Ángel, Gutiérrez, Martha Elba, Figueroa, Fausto.

"Uso de la información y su impacto educativo: la instrucción bibliográfica y su importancia”, en Reencuentro, núm. 21 (Mar. 1998), p. 20-28.

Association of College and Research Libraries (ALA). "Objetivos de formación para la alfabetización en información : un modelo de declaración para bibliotecas universitarias". Traducción de Cristóbal Pasadas Ureña, en Boletín de la Asociación andaluza de Bibliotecarios. No. 68 (Sep., 2001);p. 67-90. [Artículo en Internet] en http://www.aab.es. [Consulta: 2003-11-04].

Bernhard, Paulette. "La formación en el uso de la información : una ventaja en la enseñanza superior. Situación actual”, en Anales de Documentación. España. No. 5 (2002); pp. 409-435. 
Bibliotecas Públicas y escolares. Bogotá : Fundalectura, 2001. 186 p.

Bopp, Richar, Smith, C. Introducción general al servicio de consulta : libro de texto para el estudiante de bibliotecología y manual para el bibliotecario de consulta. Trad. Carlos C. Compton García. México : UNAM, CUIB, 2000.

Córdoba González, Saray. "La formación de usuarios con métodos participativos para estudiantes universitarios", en Desarrollo de habilidades informativas en instituciones de educación superior. Editado por Jesús Lau y Jesús Cortés. Ciudad Juárez: Universidad Autónoma de Ciudad Juárez, 2000; p. 15-24.

Cornella, Alfons. La cultura de la información como institución previa a la sociedad de la información. en:

www.infonomics.net/cornella/aprats.pdf [Consulta: 2003-01-10]

Crochik, José León. "La pseudoformación y la conciencia ilusionada", en Revista Educación y Pedagogía. Medellín. Vol. 12, no. 26-27 (Ene.-Ago., 2000); p. 159.

Cunningham, Nancy A. "Collaborating with faculty : do they understand us? Do we understand them? Do we share the same objectives?", en Desarrollo de habilidades informativas en instituciones de educación superior. Editado por Jesús Lau y Jesús Cortés. Ciudad Juárez : Universidad Autónoma de Ciudad Juárez, 2000; p. 9-14.

Díaz Monsalve, Ana Elsy, Quiroz Posada, Ruth Elena. "Reflexiones teóricas sobre la relación entre la pedagogía y la didáctica", en Lecciones Inaugurales 2002, Revista Educación y Pedagogía. Medellín. Separata No. 32 (Jun. 2002); p. 32.

Entrevista. Uriel Lozano Rivera, Luis Eduardo Villegas Puerta. Entrevista elaborada por Yicel Giraldo G. y Edilma Naranjo V. Medellín, 26 de mayo de 2004. $120 \mathrm{~min}$.

Flórez Ochoa, Rafael. Hacia una pedagogía del conocimiento. Santafé de Bogotá : McGraw-Hill, 1994; p. 210.

Gadamer, Hans-Gerg. Verdad y método. Salamanca: Ediciones Sígueme, $1991.10 \mathrm{p}$.

Gallego Escobar, Carlos, Villegas Villegas, Nelly y Bernal Ocampo, Diana. Formación de lectores autónomos, un problema que trasciende a la escuela. Medellín: [S. n.], 1998. Tesis (Magister en Educación). Universidad de Antioquia, Facultad de Educación, 1998.

Gómez Sustaíta, Rocío. La enseñanza de las habilidades informativas. [Artículo en Internet] en http://www.uag.mx/Nexo/mar01/laense.htm, 2001. [Consulta: 2003-09-30]

Gramsci, A. Cuadernos de la cárcel. México : Era, 1981. 352 p.

Guinchat, Claire, Menou, Michael. Introducción general a las ciencias y técnicas de la información y documentación / Claire Guinchat y Michael Menou. 2. ed. cor. y aum. Por Marie- France Blanquet. París: UNESCO, 1993.

Hegel, Guillermo Federico. Filosofía de la historia. Barcelona : Zeus, 1971. $268 \mathrm{p}$. 
Herder, Johann Gottfried von. Ideas para una filosofía de la historia de la bumanidad. 1. ed. Buenos Aires : Losada, 1959. 701 p.

Hernández Salazar, Patricia. "Formación de usuarios : modelo para diseñar programas sobre el uso de tecnologías de información en instituciones de educación superior", en Documentación de las Ciencias de la Información. Madrid. No. 24 (2001); p. 151-179.

- - L L formación de usuarios de la información en instituciones de educación superior. México : UNAM, Centro Universitario de Investigaciones Bibliotecológicas, 1998. 76 p.

——. Formación en el uso de información: la instrucción computarizada como alternativa. México: [s. n.], 1996. Tesis (Maestra en Bibliotecología) Universidad Nacional Autónoma de México, Facultad de Filosofía y Letras.

Honore, Bernard. Para una teoría de la formación: dinámica de la formatividad. Madrid: Narcea, 1980.

Humboldt, Wilhelm von. Sobre la diversidad de la estructura del lenguaje bumano y su influencia sobre el desarrollo espiritual de la bumanidad. Barcelona: Anthropos, 1990. 435 p.

IFLA. Manifiesto IFLA/UNESCO sobre la biblioteca escolar. Ottawa: Nacional Library of Canada, 1999. en Servicio de Bibliotecas públicas: directrices IFLA/UNESCO para el desarrollo / International Federation of Library Associations and Institutions. Bogotá: Fundalectura, 2002.

IFLA/FAIFE. Declaración de la IFLA sobre las bibliotecas y la libertad intelectual. La Haya : IFLA : FAIFE, 1999.

IFLA, Sección de Bibliotecas Públicas. Manifiesto IFLA/unesco sobre la Biblioteca Pública. La Haya: IFLA, 1995. [Documento en Internet]. en

http://www.ifla.org/VII/s8/unesco.manif.htm [Consulta: 2004-02-25]

Kant, Immanuel. Conflicto de las facultades. 1. ed. Buenos Aires: Losada, 1963. $151 \mathrm{p}$.

La Instrucción de usuarios ante los nuevos modelos educativos / Editado por Jesús Lau y Jesús Cortés. Ciudad Juárez: Universidad Autónoma de Ciudad Juárez, 2000. 160 p.

Lau, Jesús, Cortés Vera, Jesús. La agenda rezagada: la formación de usuarios de sistemas de información. [Artículo en Internet] en http://www.uacj.mx/dia/Cursos/docsdoc16.htm [Consulta:2002-08-01]

López de Prado, Rosario. Información bibliográfica : [Documento en Internet] en

www.geocities.com/zaguan2000/414.html Última revisión: 24 de abril de 2000, [Consulta : 2003-11-13].

Naranjo Vélez, Edilma. El bibliotecólogo como promotor de la lectura y sus bases pedagógicas. México : La autora, 2003. 251 h. Tesis (Maestra en Bibliotecología). Universidad Nacional Autónoma de México, Facultad de Filosofía y Letras, 2003.

Naranjo Vélez, Edilma, Álvarez Zapata, Didier. Desarrollo de habilidades informativas : una forma de animar a leer. Medellín : Universidad de Antioquia, Escuela Interamericana de Bibliotecología, 2003. 74 p. 
Naranjo Vélez, Edilma, Rendón Giraldo, Nora Elena. "Explorando el panorama de la formación de usuarios de la información", en Revista Interamericana de Bibliotecología. Medellín. Vol. 26, no. 2 (Jul. - Dic., 2003); p. 13-37.

Normas sobre alfabetización informativa en educación superior: declaratoria. Jesús Cortés... [Et al.]. en Tercer Encuentro sobre Desarrollo de Habilidades Informativas. Ciudad Juárez, 2002 (segundo borrador). Disponible en Internet en:

http://www.nclis.gov/libinter/infolitconf\&meet/JesusLauInfolitArticle-Spanish.pdf [Consulta : 2003-11-05]

Núñez Paula, Israel Adrián. "Guía metodológica para el estudio de las necesidades de formación e información de los usuarios o lectores", en ACIMED. Vol. 5, no. 3 (Sep.-Dic., 1997). 12 h. [Documento en Internet] en :

www.geocities.com/crachilcl/servicios.htm [Consulta: 2003-11-13]

Quiceno Castrillón, Humberto. "Rousseau y el concepto de Formación”, en Revista Educación y Pedagogía. Universidad de Antioquia. Medellín. No. 14-15 (1995); p. 87.

Real Academia Española. Diccionario de la lengua española. 2 ed. Madrid: Real Academia Española, 2001.

Ríos Acevedo, Clara Inés. "Un acercamiento al concepto de formación en Gadamer", en Revista Educación y Pedagogía. Medellín. Vol. 7, no. 14-15 (Jul. 1995 - Jul. 1996); p. 11-37.

Rousseau, Juan Jacobo. El Emilio o de la educación. México: Porrúa, 1986. $189 \mathrm{p}$.

Seminario Latinoamericano sobre formación de usuarios de la información y los estudios de usuarios / coord. Patricia Hernández Salazar. México : UNAM, Centro Universitario de Investigaciones Bibliotecológicas, $1997.85 \mathrm{p}$.

Servicio de Bibliotecas públicas : directrices IFLA/UNESCO para el desarrollo / International Federation of Library Associations and Institutions. Bogotá: Fundalectura, 2002.

Sharpless Smith, Susan. Library instruction on the web. en Webbased instruction: a guide for libraries / Susan Sharpless Smith. Chicago : American Library Association, 2001; p. 5-14.

Simôes Francisco, María de Fátima. "La filosofía de la educación de Rousseau. Una propuesta de relectura del Emilio", en Revista Educación y Pedagogía. Medellín. Vol. 12, no. 26-27 (Ene. - Ago., 2000); p. 65-96.

Tardon, Eugenio. Bibliotecas universitarias: concepto y función. [Documento en Internet]. en

http://www.ucm.es/BUCM/pruebas/apuntes/bu01.pdf [Consulta: 2004-07-15]

Tocatlian, Jacques. "Formación de usuarios de la información: programas, problemas y perspectivas", en Boletín de la UNESCO para las Bibliotecas. París. Vol. 32, no. 6 (Nov. - Dic., 1978); p. 382-390. 
Verdugo Sánchez, José Alfredo. "Hacia un concepto de formación de usuarios y propuesta de un programa", en Investigación Bibliotecológica": archivonomía, bibliotecología e información. México. Vol. 7, no. 15 (Jul. - Dic., 1993)

Vierhaus, Rudolf. "Formación = Bildung". Traducido por Juan Guillermo Gómez García, en Revista Educación y Pedagogía. Medellín. $2^{a}$ época, Vol. 14 (May. - Ago., 2002); separata.

Wilson, T. D. Directrices para el planeamiento y la ejecución de un plan nacional de formación y enseñanza para el uso de la información. París : Unesco, 1980. 54 p.

."El modelado orientado al usuario : una perspectiva global", en Anales de Documentación. Murcia. No. 2 (1999); p. 85-94. 